Pacific Journal of Mathematics

MAPPINGS BY PARALLEL NORMALS PRESERVING 


\section{MAPPINGS BY PARALLEL NORMALS PRESERVING PRINCIPAL DIRECTIONS}

\section{DIMITRI KOUTROUFIOTIS}

Two smooth surfaces $S, \bar{S}$ with positive Gaussian curvature and with the same closed hemisphere as spherical image can be mapped onto each other by parallel normals. It is assumed, in addition, that principal directions at every point on $S$ are mapped into principal directions at the image point on $\bar{S}$. Let $k_{i}(=1,2)$ be the principal curvatures of $S, \bar{k}_{i}$ the corresponding principal curvatures of $\bar{S}$. Via the spherical image mapping, one may consider the function $\varphi=\left(k_{1}^{-1}-\bar{k}_{1}^{-1}\right)$. $\left(k_{2}^{-1}-\bar{k}_{2}^{-1}\right)$ as being defined on the unit sphere $\Sigma$. We show: If $\varphi$ does not change sign and appropriate boundary conditions are satisfied, then $S$ differs from $\bar{S}$ by a translation. Since the spherical image mapping always preserves principal directions, one obtains in particular characterizations of the hemisphere. Further results for ovaloids $S, \bar{S}$ within this class of mappings: If $\bar{k}_{1} \geqq k_{1}, \bar{k}_{2} \geqq k_{2}$ everywhere, then a translate of $\bar{S}$ fits inside $S$; if $S$ and $\bar{S}$ have the same total mean curvature, then $\int_{\Sigma} \varphi d \omega \leqq 0$ with equality if and only if $S$ is a translate of $\bar{S}$.

Let $S, \bar{S}$ be smooth, oriented surfaces with positive Gaussian curvature in fixed position in $E^{3}$. Assume that their spherical images are simple and coincident, so that they can be mapped diffeomorphically onto each other by equal normals. We impose the additional condition that, under this standard mapping-which we shall henceforth call the normal mapping-, principal directions are preserved, i.e., that at every point on $S$ there exists a pair of principal directions which are mapped into principal directions at the image point on $\bar{S}$. The normal mapping between surfaces of revolution with parallel axes of rotation has this property. If $S$ is an ovaloid and $\bar{S}$ a sphere, then the normal mapping again certainly preserves principal directions. Further "trivial" examples are furnished by pairs of homothetic surfaces or pairs of surfaces which are parallel in the classical sense of Steiner. This last class has been investigated in [9]. Here various geometric conclusions will be drawn from the existence of such a mapping in the large in conjunction with given boundary conditions and inequalities connecting the principal curvatures of $S, \bar{S}$ at corresponding points. In particular, we shall obtain congruence theorems, characterizations of the sphere and statements about relative size. These geometric results are in part generalizations of our geometric results in [8]. They are for the most part direct consequences of the 
analytic results in that paper which we will review briefly in the next section. However, Proposition 1 and Theorem 3 are new and belong properly the analytic results in [8].

2. We denote by $\Sigma$ the unit sphere in $E^{3}$, centered at the origin. We choose parameters $\left(u^{1}, u^{2}\right)$ so that the unit vector $\vec{n}\left(u^{1}, u^{2}\right)$ describing $\Sigma$ is analytic and the unit normal of $\Sigma$ at the point $\vec{n}$ is $-\vec{n}$ (interior normal). We will use the summation convention throughout this paper; thus $n_{i k} d u^{i} d u^{k}$, where $n_{i k}=\vec{n}_{i} \cdot \vec{n}_{k}$, denotes the standard metric on $\Sigma$. The discriminant tensor $\varepsilon^{i k}$ is defined by

$$
\varepsilon^{11}=\varepsilon^{22}=0, \varepsilon^{21}=-\varepsilon^{12}=\left(\operatorname{det} n_{i k}\right)^{-1 / 2} .
$$

Let $f, \bar{f}$ be real-valued functions of class $C^{2}$ on a subset of $\Sigma$. We introduce the classical invariant differential operators on $\Sigma$. The scalar product of the gradients of $f$ and $\bar{f}$ :

$$
\nabla(f, \bar{f})=n^{i k} \frac{\partial f}{\partial u^{i}} \frac{\partial \bar{f}}{\partial u^{k}},
$$

where $n^{i k}=\varepsilon^{i \alpha} \varepsilon^{k \beta} n_{\alpha \beta}$; the Laplace-Beltrami operator

$$
\Delta_{2} f=n^{i k} \nabla_{i k} f \text {, }
$$

where $\left(\nabla_{i k} f\right)$ is the tensor of the second covariant derivatives of $f$. We set $\nabla^{i k} f=\varepsilon^{i \alpha} \varepsilon^{k \beta} \nabla_{\alpha \beta} f$ and define

$$
\Delta_{22}(f, \bar{f})=\nabla_{i k} f \nabla^{i k} \bar{f}=\Delta_{22}(\bar{f}, f) .
$$

Note that $\Delta_{22}(f, \bar{f})$ is twice the Hessian of $f$.

Our main concern will be with the operator $M$ defined by

$$
M[f, f]=\Delta_{22}(f, \bar{f})+f \Delta_{2} \bar{f}+\bar{f} \Delta_{2} f+2 f \bar{f} .
$$

We review some of its theory. See [8] and [9] for more details. $M$ is clearly bilinear and symmetric. If $L$ is the restriction to $\Sigma$ of a linear function defined on $E^{3}$, one verifies directly that, for arbitrary $f, M[f, L]=0$. Further, for any $C^{2}$ functions $h, f, \bar{f}$ on $\Sigma$ we have (Minkowski):

$$
\int_{\Sigma} h M[f, \bar{f}] d \omega=\int_{\Sigma} \bar{f} M[f, h] d \omega .
$$

See e.g., [13], p. 244 for a proof. In particular,

$$
\int_{\Sigma} L M[f, \bar{f}] d \omega=0 \text {, for any } L \text { on } \Sigma \text {. }
$$

Now let $g$ be a given continuous function on $\Sigma$. We obtain from (2.2) a necessary condition for the existence of global $C^{2}$ solutions 
of the nonlinear equation

$$
M[f, f]=g,
$$

namely,

$$
\int_{\Sigma} g L d \omega=0, \text { for all linear function } L \text { on } \Sigma \text {. }
$$

Condition (2.4) is also sufficient for solvability in case $g$ is strictly positive and sufficiently smooth on $\Sigma$ ([10], p. 381, Theorem 4).

If $g \equiv 0$, then, of course, all linear functions satisfy (2.3). A global converse is also true [8]:

THEOREM 1. Let $f$ be of class $C^{2}$ on a closed hemisphere and satisfy $M[f, f]=0$ there. Then $f$ is linear (i.e., the restriction to the closed hemisphere of a linear function in $E^{3}$ ).

In case $g<0$, condition (2.4) is not sufficient for solvability. In fact, we have:

Proposition 1. There exists no function $f$, defined and of class $C^{2}$ on $\Sigma$, satisfying $M[f, f]<0$ everywhere.

Thus, for example, $M[f, f]=-1$ is not solvable, although (2.4) is satisfied.

Proof. Assume such an $f$ exists. Define the vector-valued function $\vec{x}\left(u_{1}, u_{2}\right)$ of class $C^{1}$ by

$$
\vec{x}=f \vec{n}+\nabla(f, \vec{n}),
$$

where $\nabla(f, \vec{n})$ is defined as in (2.0) with the vector $\vec{n}$ replacing $\bar{f}$. One has

$$
\frac{\partial \vec{x}}{\partial u^{i}} \cdot \vec{n}=0
$$

and, using the Gauss equations for $\Sigma$, one computes

$$
\begin{aligned}
\frac{\partial \vec{x}}{\partial u^{i}} \cdot \frac{\partial \vec{n}}{\partial u^{j}}=f n_{\imath j}+\nabla_{i j} f, & \left(\frac{\partial \vec{x}}{\partial u^{1}} \times \frac{\partial \vec{x}}{\partial u^{2}}\right) \cdot\left(\frac{\partial \vec{n}}{\partial u^{1}} \times \frac{\partial \vec{n}}{\partial u^{2}}\right) \\
= & \left(\frac{\partial \vec{x}}{\partial u^{1}} \cdot \frac{\partial \vec{n}}{\partial u^{1}}\right)\left(\frac{\partial \vec{x}}{\partial u^{2}} \cdot \frac{\partial \vec{n}}{\partial u^{2}}\right)-\left(\frac{\partial \vec{x}}{\partial u^{1}} \cdot \frac{\partial \vec{n}}{\partial u^{2}}\right)^{2} \\
= & \frac{1}{2}\left(\operatorname{det} n_{i k}\right) M[f, f] \neq 0 .
\end{aligned}
$$


The mapping (2.5) of $\Sigma$ into $E^{3}$ defines a compact, oriented surface $S$ of class $C^{1}$, with normal $\pm \ddot{n}$ of class $C^{\omega}$, so that $S$ is in fact of class $C^{2}$ ([4], p. 307). From (2.6) we deduce that $S$ is a regular differential-geometric surface and, since it is of class $C^{2}$, its Gaussian curvature $K$ is defined and continuous. Now, by a classical formula of differential-geometry,

$$
\frac{\partial \vec{n}}{\partial u^{1}} \times \frac{\partial \vec{n}}{\partial u^{2}}=K\left(\frac{\partial \vec{x}}{\partial u^{1}} \times \frac{\partial \vec{x}}{\partial u^{2}}\right) .
$$

Since $M[f, f]<0$, one would therefore have, from (2.6), a compact $C^{2}$ surface in $E^{3}$ with negative curvature throughout, an impossibility since every such surface possesses points of positive curvature.

A statement can also be made in the case $g \leqq 0[8]$ :

LemMa 1. Let $f$ be of class $C^{2}$ on a closed hemisphere and satisfy $M[f, f] \leqq 0$ there; then $f$ vanishes at some point on the bounding great circle.

THEOREM 2. Let $f$ be of class $C^{\text {p }}$ on a closed homisphere and satisfy $M[f, f] \leqq 0$ there and $f=$ constant on the bounding great circle. Then $f$ is linear.

The proof of this theorem makes use of the preceding lemma to conclude that $f=0$ on the bounding great circle; and of Bernstein's theorem [6] concerning the growth of saddle surfaces defined over the whole plane to conclude that $M[f, f] \equiv 0$. By Theorem $1, f$ must then be linear.

In the case $g \geqq 0$ the preceding lemma does not hold. However, a slightly weaker form of Theorem 2 still holds:

THEOREM 3. Let $f$ be of class $C^{2}$ on a closed hemisphere and satisfy $M[f, f]>0$ there and $f=0$ on the bounding great circle. Then $f$ is linear.

The proof is formally the same as that of Theorem 2; we merely replace Bernstein's theorem by its companion theorem concerning the growth of convex functions defined over the whole plane ([11], p. 623, and [5] for the $C^{2}$ case): Let the function $F(x, y)$, defined and of class $C^{2}$ for all $(x, y)$, satisfy $F_{x x} F_{y y}-F_{x y}^{2} \geqq 0$ everywhere and $F(x, y)=$ $o\left(\sqrt{\left.x^{2}+y^{2}\right)}\right.$ at infinity; then $F_{x x} F_{y y}-F_{x y}^{2} \equiv 0$.

3. We shall be concerned exclusively with surface in $E^{3}$, of class $C^{3}$, having positive Gaussian curvature, oriented by "interior" normals so that the principal curvatures are positive, and mapped 
diffeomorphically onto a schlicht subset of $\Sigma$ under the normal mapping; we shall cal them strictly convex surfaces. For the sake of brevity, we shall call a surface of this type with boundary and a closed hemisphere as spherical image strictly convex hemispherical. Every enveloping cylinder of an ovaloid (compact surface, $K>0$ ) divides it into two strictly convex hemispherical surfaces with common boundary. When we consider pairs of such surfaces in the sequel, we shall always assume implicitly that their spherical images coincide.

If the surface $S$ is mapped with preservation of principal directions onto the surface $\bar{S}$, we denote by $\bar{k}_{i}(i=1,2)$ the (principal) curvature of the direction on $\bar{S}$ which is the image of the principal direction on $S$ with curvature $k_{i}$.

If the strictly convex surface $S$ of class $C^{3}$ has "interior" normal $\vec{\nu}$ at the point with position vector $\vec{x}$, we define the support function $p$ of $S$ by $p=-\vec{x} \cdot \dot{\nu}$. We will view $p$ as defined on the spherical image of $S$. By the inverse function theorem, $p$ is of class $C^{2}$.

LEMMA 2. Let $S, \bar{S}$ be strictly convex surfaces with support functions $p, \bar{p}$, mapped diffeomorphically onto each other by the normal mapping. Assume that principal directions are preserved at an interior point with normal $\vec{\nu}_{0}$. Then the following equation is satisfied at $\vec{\nu}_{0}$ :

$$
\frac{1}{2} M[p-\bar{p}, p-\bar{p}]=\left(\frac{1}{k_{1}}-\frac{1}{\bar{k}_{1}}\right)\left(\frac{1}{k_{2}}-\frac{1}{\bar{k}_{2}}\right) .
$$

Proof. Using the bilinearity and symmetry of $M$, we obtain:

$$
M[p-\bar{p}, p-\bar{p}]=M[p, p]+M[\bar{p}, \bar{p}]-2 M[p, \bar{p}] .
$$

On the other hand, by Weingarten's formula (see [9] for a proof), we have quite generally:

$$
M[p, p]=\frac{2}{k_{1} k_{2}}, M[\bar{p}, \bar{p}]=\frac{2}{\bar{k}_{1} \bar{k}_{2}},
$$

and by Lemma 1 of [9] we have specifically at the normal $\vec{\nu}_{0}$ :

$$
M[p, \bar{p}]=\frac{1}{k_{1} \bar{k}_{2}}+\frac{1}{k_{2} \bar{k}_{1}},
$$

and (3.1) follows.

We now apply Lemma 2 in conjunction with the theorems of $\S 1$. Note that adding a linear function to the support function of a surface amounts to a translation of the surface. From Theorem 1 we obtain immediately: 
THEOREM 1'. Let $S, \bar{S}$ be strictly convex hemispherical surfaces; assume that the normal mapping between them is a diffeomorphism which preserves principal directions. If, in addition, $\left(k_{1}-\bar{k}_{1}\right)\left(k_{2}-\right.$ $\left.\bar{k}_{2}\right)=0$, then $S$ is a translate of $\bar{S}$.

In particular, the same conclusion holds if $S, \bar{S}$ are ovaloids. Note that this theorem does not hold in the small. Consider, for example, a small piece $S$ of a strictly convex surface of revolution with the $z$-axis as axis of rotation. Take the meridian curve $C$ on the $(z, x)$-plane and translate it some distance in the $x$-direction: One obtains a curve $\bar{C}$ wich one then rotates around the $z$-axis to obtain a surface of revolution $\bar{S}$. The hypotheses of Theorem $1^{\prime}$ are satisfied for $S$ and $\bar{S}$, the conclusion is however false.

Proposition 1 applied to our context says that the inequality $\left(k_{1}-\bar{k}_{1}\right)\left(k_{2}-\bar{k}_{2}\right)<0$ is not possible everywhere on $\Sigma$ if the normal mapping between the ovaloids $S, \bar{S}$ preserves principal directions.

The geometric version of Theorem 2 via Lemma 2 reads:

THEOREM 2'. Assume that the normal mapping between the strictly convex hemispherical surfaces $S, \bar{S}$ preserves principal directions. If $\left(k_{1}-\bar{k}_{1}\right)\left(k_{2}-\bar{k}_{2}\right) \leqq 0$ everywhere and $p-\bar{p}=$ constant on the bounding great circle of their common spherical image, then the surfaces differ by a translation.

The condition " $p-\bar{p}=$ constant on the boundary" means that the enveloping cylinders of $S, \bar{S}$ touching along their respective boundaries are, after a possible translation, parallel surfaces in the classical sense. Thus, if for example the enveloping cylinders are circular or if the surfaces touch along their common boundary, we may assume that $p-\bar{p}=$ constant.

If we take for $\bar{S}$ a closed hemisphere centered at the origin, we conclude that if a strictly convex hemispherical surface has a circular enveloping cylinder along its boundary and satisfies $\left(k_{1}-c\right)\left(k_{2}-c\right) \leqq$ 0 for some constant $c$, it must be a hemisphere [8]. From this we deduce in particular:

COROLLARY. Let $F(x, y)$ be a real-valued function, defined for $x \geqq y \geqq 0$, monotonic in the same sense in both arguments and not identically zero on any open set. Let $S$ be a strictly convex hemispherical surface, with circular enveloping cylinder along its boundary and at least one umbilic. If $F\left(k_{1}, k_{2}\right)=0$ everywhere $\left(k_{1} \geqq k_{2}\right)$, then $S$ is a hemisphere.

The proof is standard. Let $P_{0}$ be the umbilic and let $k_{1}\left(P_{0}\right)=$ 
$k_{2}\left(P_{0}\right)=c$. If for any point $P \neq P_{0}$ we had $k_{1}(P)>c$ and $k_{2}(P)>c$, then the equation $F\left(k_{1}, k_{2}\right)=0$ and the monotonicity of $F$ would imply that $F(x, y)$ is identically zero on the set $c \leqq x \leqq k_{1}(P), c \leqq y \leqq k_{2}(P)$ in contradiction to our hypothesis. The possibility $k_{1}(P)<c$ and $k_{2}(P)<c$ is discarded in the same fashion. Therefore $\left(k_{k_{1}}-c\right)\left(k_{2}-c\right) \leqq$ 0 everywhere, hence $S$ must be a hemisphere.

The existence of an umbilic on $S$ in this coroliary is certain if, for example, the enveloping cylinder touches $S$ along a circle. Note that no smoothness is required of $F$. If, however, $F$ is of class $C^{2}$, then this characterization of hemispheres follows from a theorem of Aleksandrov ([1], p. 349).

From Theorem 3 we obtain:

THEOREM 3'. Let $S, \bar{S}$ be strictly convex hemispherical surfaces, diffeomorphic under the normal mapping which should preserve principal directions, and with enveloping cylinders along their boundaries which are translates of one another. If , in addition, $\left(k_{1}-\bar{k}_{1}\right)\left(k_{2}-\right.$ $\left.\bar{k}_{2}\right) \geqq 0$, then $S$ is a translate of $\bar{S}$.

In particular, a strictly convex hemispherical surface with $\left(k_{1}-\right.$ $c)\left(k_{2}-c\right) \geqq 0$, whose enveloping cylinder touching along the boundary is circular of radius $c^{-1}$, is a hemisphere.

REMARK. If we replace the inequality in Theorem $3^{\prime}$ by the stronger hypothesis that $k_{1} \geqq \bar{k}_{1}$ and $k_{2} \geqq \bar{k}_{2}$ everywhere, then the conclusion holds even without the assumption that the normal mapping preserves principal directions. In fact, we have more generally: If the strictly convex hemispherical surfaces $S, \bar{S}$ have identical enveloping cylinders along their boundaries and their Gaussian curvatures satisfy $K \geqq \bar{K}$ at all points with the same normal, then they are identical. The proof is contained, in its essential, in [1], p. 348; the support functions $p, \bar{p}$ satisfy respectively the partial differential equations $M[f, f]=2 K^{-1}$ and $M[f, f]=2 \bar{K}^{-1}$ which are elliptic because $K>0$ and $\bar{K}>0$. It follows that the operator $M$ is elliptic with respect to all the functions $t p+(1-t) \bar{p}, t \in[0,1]$, which are also support functions of strictly convex surfaces. After an appropriate translation in the direction of their common enveloping cylinder, we may assume that $\bar{S}$ lies below $S$ and touches $S$ "from the inside" at a point (which may be a boundary point). We then have $M[p, p] \leqq$ $M[\bar{p}, \bar{p}]$ and $p \geqq \bar{p}$, therefore, by the maximum principle ([12]), $S$ and $\bar{S}$ coincide.

A result concerning the relative size of pairs of ovaloids within our class is this: 
THEOREM. Assume that the normal mapping between the ovaloids $S, \bar{S}$ preserves principal directions and that $\bar{k}_{1} \geqq k_{1}, \bar{k}_{2} \geqq k_{2}$ at all corresponding points. We can then translate $\bar{S}$ so that it lies entirely within $S$.

Proof. For any $\varepsilon>0$, consider the ovaloid $S_{\varepsilon}$, parallel to $S$, obtained by moving along every outward normal of $S$ a distance $\varepsilon$. If $p$ is the support function of $S$ and $k_{i}^{-1}$ its radii of curvature, $S_{\varepsilon}$ has support function $\varepsilon+p$ and radii of curvature $\varepsilon+k_{i}^{-1}$ ([3], p. 273). Since the normal mapping between parallel surfaces preserves principal directions, so does the normal mapping between $\bar{S}$ and $S_{\varepsilon}$. Therefore, by Lemma 2,

$$
\begin{aligned}
\frac{1}{2} M[ & \varepsilon+p-\bar{p}, \varepsilon+p-\bar{p}] \\
& =\left[\varepsilon+\left(\frac{1}{k_{1}}-\frac{1}{\bar{k}_{1}}\right)\right]\left[\varepsilon+\left(\frac{1}{k_{2}}-\frac{1}{\bar{k}_{2}}\right)\right]>0 .
\end{aligned}
$$

Set $\varepsilon+p-\bar{p}=f$. As in the proof of Proposition 1, we consider the $C^{1}$-, compact, oriented surface $S^{*}$ defined by (2.5), where $\vec{n}$ describes the unit sphere oriented by interior normals $-\vec{n}$. Since $M[f$, $f] \neq 0$, equation (2.6) implies that $S^{*}$ is regular, with normal $-\vec{n}$ and hence of class $C^{2}$. The support function of $S^{*}$ is $\vec{x} \cdot \vec{n}=f$. The Gaussian curvature of $S^{*}$ is therefore $2(M[f, f])^{-1}$ which is positive throughout $S^{*}$. By Hadamard's theorem, $S^{*}$ is simple and convex and the mapping of $\Sigma$ onto $S^{*}$ defined by (2.5) is the inverse of the spherical-image mapping of $S^{*}$, which preserves orientation. Hence, $-\vec{n}$ is the interior normal of $S^{*}$ and we may translate $S^{*}$ so that the origin lies in its interior and the new support function is positive. Thus, $f+L>0$ for a certain linear function $L$, or: $\varepsilon+p+L>\bar{p}$; which means that, after a possible translation, $\bar{S}$ lies inside $S_{\varepsilon}$. Since this is true for every $\varepsilon>0$, it must also be true for $S=S_{0}$, which proves the theorem.

In particular, we may take one or the other of the two surfaces in this theorem to be a sphere. It follows that if the maximal principal curvature $k_{1}$ of an ovaloid $S$ satisfies $k_{1} \leqq c$, then a sphere of radius $c^{-1}$ fits inside $S$; and if the minimal principal curvature $k_{2}$ of $S$ satisfies $k_{2} \geqq c$, then $S$ fits inside a sphere of radius $c^{-1}$. Of course these special cases are well-known and much more can be said about them [7].

4. Some information about normal mappings which preserve principal direction can also be obtained with the aid of integral formulas. 
Consider a $C^{2}$ function $f$ on the unit sphere $\Sigma$. By Green's theorem,

$$
\int_{\Sigma}\left[\nabla(f, f)+f \Delta_{2} f\right] d \omega=0
$$

Therefore,

$$
\int_{\Sigma} f\left(\Delta_{2} f+2 f\right) d \omega=\int_{\Sigma}\left[2 f^{2}-\nabla(f, f)\right] d \omega
$$

Wirtinger's lemma ([2], p. 108) states that if

$$
\int_{\Sigma} f d \omega=0, \text { then } \int_{\Sigma}\left[2 f^{2}-\nabla(f, f)\right] d \omega \leqq 0
$$

with equality holding if and only if $f$ is linear.

Consider now an ovaloid $S$, with area element $d A$, total area $A$, mean curvature $H$, total mean curvature $M$, etc. We have, as is well-known:

$$
M=\int_{S} H d A=\int_{\Sigma} p d \omega, \quad \Delta_{2} p+2 p=\frac{2 H}{K}
$$

We note in passing an immediate consequence of the above, valid for arbitrary normal mappings (take $f=p-\bar{p}$ ):

Proposition 2. Assume that the ovaloids $S, \bar{S}$ have the same total mean curvature. Then

$$
\int_{\Sigma}(p-\bar{p})\left(\frac{H}{K}-\frac{\bar{H}}{\bar{K}}\right) d \omega \leqq 0,
$$

with equality holding if and only if $S$ is a translate of $\bar{S}$.

In particular, we may take for $\bar{S}$ a sphere centered at the origin, with radius $(4 \pi)^{-1} M$. Then $M=\bar{M}$ and multiplying out in (4.2) we obtain Minkowski's inequality:

$$
4 \pi A \leqq M^{2},
$$

with equality if and only if $S$ is a sphere.

If at all pairs of points of $S, \bar{S}$ with the same normal we have $H K^{-1}=\bar{H} \bar{K}^{-1}$, then automatically $\bar{M}=M$ and we obtain as corollary of Proposition 2 the theorem of Christoffel: $S$ is a translate of $\bar{S}$.

Note, from the definition of $M$, that $M[f, 1]=\Delta_{2} f+2 f$. From (2.1), setting $f=\bar{f}, h=1$, we obtain:

$$
\int_{\Sigma} M[f, f] d \omega=\int_{\Sigma} f M[f, 1] d \omega=\int_{\Sigma} f\left(\Delta_{2} f+2 f\right) d \omega .
$$


From (4.1) and Wirtinger's lemma we conclude that if

$$
\int_{\Sigma} f d \omega=0, \text { then } \int_{\Sigma} M[f, f] d \omega \leqq 0,
$$

with equality if and only if $f$ is linear.

Consider now two ovaloids of the type under investigation. Setting $f=p-\bar{p}$ and using Lemma 2, we obtain

Proposition 3. Let the normal mapping between the ovaloids $S, \bar{S}$ preserve principal directions. If, in addition, $S$ and $\bar{S}$ have the same total mean curvature, then

$$
\int_{\Sigma}\left(\frac{1}{k_{1}}-\frac{1}{\bar{k}_{1}}\right)\left(\frac{1}{k_{2}}-\frac{1}{\bar{k}_{2}}\right) d \omega \leqq 0,
$$

with equality holding if and only if $S, \bar{S}$ differ by a translation.

\section{REFERENCES}

1. A. D. Aleksandrov, Uniqueness theorems for surfaces in the large I, Amer. Math. Soc. Translations, (series 2) 21, 341-354.

2. W. Blaschke, Kreis und Kugel, 2. Auflage, Berlin, 1956.

3. L. P. Eisenhart, An Introduction to Differential Geometry, Princeton, 1947.

4. P. Hartman and A. Wintner, On the third fundamental form of a surface, Amer. J. Math., 75 (1953), 298-334.

5. — On isometric immersions in euclidean space of manifolds with nonnegative sect?onal curvatures II, Amer. Math. Soc. Transactions, 147 (1970), 529-540. 6. E. Hopf, On S. Bernstein's theorem on surfaces $z(x, y)$ of nonpositive curvature, Proc. Amer. Math. Soc., 1 (1950), 80-85.

7. D. Koutroufiotis, On Blaschke's rolling theorems, Arch. Math., 23 (1972), 655-660.

8. - On a conjectured characterization of the sphere, Math. Ann., 205 (1973), 211-217.

9. - A characterization of parallel ovaloids, to appear.

10. L. Nirenberg, The Weyl and Minkowski problems in differential geometry in the large, Comm. Pure Appl. Math., 6 (1953), 337-394.

11. R. Sacksteder, On hypersurfaces with no negative sectional curvatures, Amer. J. Math., 82 (1960), 609-630.

12. J. Serrin, Nonlinear elliptic equations of second order, Amer. Math. Soc. Summer Institute on Partial Differential Equations, Berkeley, 1971.

13. J. J., Stoker, On the uniqueness theorems for the embedding of convex surfaces in three-dimensional space, Comm. Pure Appl. Math., 3 (1950), 213-257.

Received March 5, 1973.

University of California, Santa Barbara 


\section{PACIFIC JOURNAL OF MATHEMATICS}

\section{EDITORS}

RICHARD ARENS (Managing Editor)

University of California

Los Angeles, California 90024

R. A. Beaumont

University of Washington

Seattle, Washington 98105
J. DugundJI

Department of Mathematics University of Southern California Los Angeles, California 90007

D. Gilbarg AND J. Milgram Stanford University

Stanford, California 94305

\section{ASSOCIATE EDITORS}
E. F. BECKENBACH
B. H. NeUmanN
F. WOLF
K. YOSHIDA

\section{SUPPORTING INSTITUTIONS}

UNIVERSITY OF BRITISH COLUMBIA CALIFORNIA INSTITUTE OF TECHNOLOGY

UNIVERSITY OF CALIFORNIA

MONTANA STATE UNIVERSITY

UNIVERSITY OF NEVADA

NEW MEXICO STATE UNIVERSITY

OREGON STATE UNIVERSITY

UNIVERSITY OF OREGON

OSAKA UNIVERSITY
UNIVERSITY OF SOUTHERN CALIFOF

STANFORD UNIVERSITY

UNIVERSITY OF TOKYO

UNIVERSITY OF UTAH

WASHINGTON STATE UNIVERSITY UNIVERSITY OF WASHINGTON

AMERICAN MATHEMATICAL SOCIET NAVAL WEAPONS CENTER 


\section{Pacific Journal of Mathematics}

\section{Vol. 54, No. 1 \\ May, 1974}

Ralph K Amayo, Engel Lie rings with chain conditions ..................

Bernd Anger and Jörn Lembcke, Hahn-Banach type theorems for hypolinear

functionals on preordered topological vector spaces ..................

Gregory Frank Bachelis and Samuel Ebenstein, On $\Lambda(p)$ sets ................

Harvey Isaac Blau, Indecomposable modules for direct products of finite

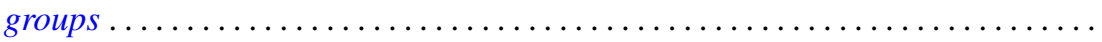

Larry Eugene Bobisud and James Calvert, Singular perturbation of a

time-dependent Cauchy problem in a Hilbert space ................

Walter D. Burgess and Robert Raphael, Abian's order relation and orthogonal

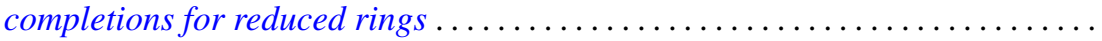

James Diederich, Representation of superharmonic functions mean continuous at

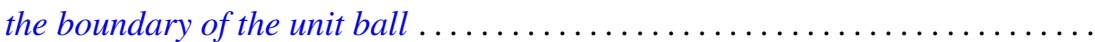

Aad Dijksma and Hendrik S. V. de Snoo, Self-adjoint extensions of symmetric

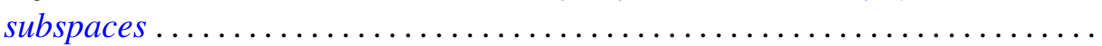

Gustave Adam Efroymson, A Nullstellensatz for Nash rings . . . . . . . . . . . . .

John D. Elwin and Donald R. Short, Branched immersions onto compact orientable surfaces . . . . . . . . . . . . . . . . . . . . . . . . .

John Douglas Faires, Comparison of the states of closed linear

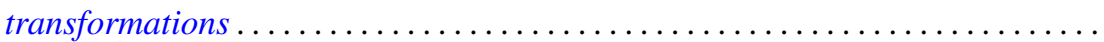

Joe Wayne Fisher and Robert L. Snider, On the von Neumann regularity of rings with regular prime factor rings .

Franklin Takashi Iha, A unified approach to boundary value problems on compact intervals

Palaniappan L. Kannappan and Che Tat $\mathrm{Ng}$, On functional equations connected with directed divergence, inaccuracy and generalized directed divergence

Samir A. Khabbaz and Elias Hanna Toubassi, The module structure of Ext $(F, T)$ over the endomorphism ring of $T$...

Garo K. Kiremidjian, On deformations of complex compact manifolds with boundary.

Dimitri Koutroufiotis, Mappings by parallel normals preserving principal

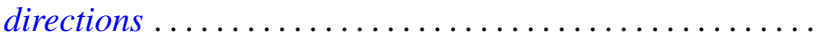

W. K. Nicholson, Semiperfect rings with abelian adjoint group

Norman R. Reilly, Extension of congruences and homomorphisms to translational hulls

Sadahiro Saeki, Symmetric maximal ideals in $M(G)$

Brian Kirkwood Schmidt, On the homotopy invariance of certain functors ...

H. J. Shyr and T. M. Viswanathan, On the radicals of lattice-ordered rings ...

Indranand Sinha, Certain representations of infinite group algebras ...

David Smallen, The group of self-equivalences of certain complexes ...

Kalathoor Varadarajan, On a certain problem of realization in homotopy

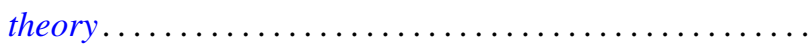

